\begin{tabular}{|c|c|}
\hline Citation & $\begin{array}{l}\text { Van der Kelen, C., Göransson, P., Pluymers, B., Desmet, W. (2014), } \\
\text { On the influence of frequency-dependent elastic properties in vibro- } \\
\text { acoustic modelling of porous materials under structural excitation } \\
\text { Journal of Sound and Vibration, } 333 \text { (24), 6560-6571. }\end{array}$ \\
\hline Archived version & $\begin{array}{l}\text { Author manuscript: the content is identical to the content of the published } \\
\text { paper, but without the final typesetting by the publisher }\end{array}$ \\
\hline Published version & http://dx.doi.org/10.1016/1.jsv.2014.07.032 \\
\hline Journal homepage & http://www.sciencedirect.com/science/article/pii/S0022460X14006294 \\
\hline Author contact & youremail xxx@xxx.kuleuven.be \\
\hline & your phone number $+32(0) 16 x x x x x x$ \\
\hline IR & https://lirias.kuleuven.be/handle/123456789/437665 \\
\hline
\end{tabular}

(article begins on next page) 


\title{
On the influence of frequency-dependent elastic properties in vibro-acoustic modelling of porous materials under structural excitation
}

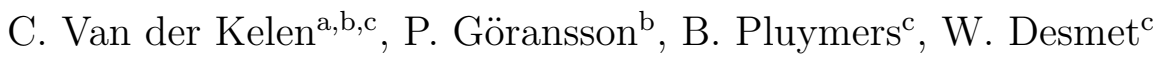 \\ ${ }^{a}$ cjfvdk@kth.se \\ ${ }^{b}$ KTH, Royal Institute of Technology, Stockholm, Sweden \\ ${ }^{c}$ K.U.Leuven, Department of Mechanical Engineering, Heverlee, Belgium
}

\begin{abstract}
The aspects related to modelling the frequency dependence of the elastic properties of air-saturated porous materials have been largely neglected in the past for several reasons. For acoustic excitation of porous materials, the material behaviour can be quite well represented by models where the properties of the solid frame have little influence. Only recently has the importance of the dynamic moduli of the frame come into focus. This is related to a growing interest in the material behaviour due to structural excitation. Two aspects stand out in connection with the elastic-dynamic behaviour. The first is related to methods for the characterisation of the dynamic moduli of porous materials. The second is a perceived lack of numerical methods able to model the complex material behaviour under structural excitation, in particular at higher frequencies. In the current paper, experimental data from a panel under structural excitation, coated with a porous material are presented. In an attempt to correlate the experimental data to numerical predictions, it is found that the measured quasi-static material parameters do not suffice for an accurate prediction of the measured results. The elastic
\end{abstract}


material parameters are then estimated by correlating the numerical prediction to the experimental data, following the physical behaviour predicted by the augmented Hooke's Law. The change in material behaviour due to the frequency-dependent properties is illustrated in terms of the propagation of the slow wave and the shear wave in the porous material.

Keywords: porous materials, elastic properties, measurements, numerical simulations, augmented Hooke's law

\section{Introduction}

Porous materials saturated with air are frequently used as passive noise absorbing components in the automotive, aeronautical and rail industries. Hand in hand with the increased importance of virtual design, there is a significant increase in the interest for numerical tools to simulate the effects of such materials. Accurate modelling of the vibro-acoustic behaviour of these materials is crucial in an early stage of the development process to obtain good noise and vibration performance in the final vehicle design. A particular challenge is to model the propagation of structurally excited waves in the porous material, and the various associated dissipation mechanisms within the material, which has been studied by several authors $[1,2,3,4]$. For a high fidelity prediction, the elastic, visco-elastic, acoustic and visco-acoustic properties of both the solid frame and the saturating fluid need to be taken into account [5]. This requires two aspects to be satisfactorily resolved, a properly characterised material model and adequate simulation techniques based on this. 
The 3D behaviour of porous materials is very complex, and current modelling techniques are not fully equipped to accurately deal with this complexity, especially at the mid and high frequency ranges. Therefore it is in engineering applications often tempting to consider some assumptions that simplify the models of these materials. Examples of such models assume that the solid frame of the material is rigid or infinitely flexible [6], giving good results for materials under acoustic excitation. Other examples are simplifications in geometry which allow for studying the 1D behaviour of the material, e.g. impedance tubes, or 1D radiation efficiency [6]. However, in the case that the 3D behaviour of finite sized porous materials under structural excitation needs to be studied, the involvement of excited bending waves and the interaction between the solid and fluid phase of the material, requires a different modelling approach than for monophasic media (solid, fluid) [7]. Supporting this, recent advances have been made in modelling structurally excited porous materials for simplified geometries, using hierarchical elements $[8,9,10]$.

An additional difficulty in accurately modelling the influence and behaviour of isotropic poroelastic materials used for vibro-acoustic purposes, is an accurate characterisation of their constitutive properties. The material's acoustic behaviour is well described with the Johnson-ChampouxAllard (JCA) model [11] when the frame may be considered motionless, by the parameters porosity, flow resistivity, tortuosity and viscous and thermal characteristic lengths, from low to high frequency. These parameters are assumed to be constant over the frequency range, and can for instance be 
determined by direct or indirect measurement $[11,12,13]$ or inverse estimation of these properties [14]. In contrast, the properties related to the frame of isotropic poroelastic materials, the Young's modulus, Poisson ratio and loss factor, are often assumed to be constant with frequency. However, previous research results indicate that these properties need to be introduced as frequency-dependent due to the visco-elasticity of the solid frame $[15,16,17,18,19,20,21,22]$. If these properties are determined at one single frequency and assumed constant over the whole frequency range, an inaccurate material model of the behaviour of the porous material may result. This was clearly shown by Rigobert et al. [1], who made a comparison between the measured and predicted response for a plate coated with a porous material. It was illustrated that the elastic properties of the material had to be modified in different frequency intervals, in order to get a good fit between measurements and predictions.

The objective of the current paper is to emphasize the necessity of including the frequency dependence of the solid frame properties in the context of structural excitation of poroelastic materials. For this purpose, new experimental data are presented involving a clamped steel plate panel excited at its boundaries, and coated with different porous materials. As a first step, the measurements are compared to the numerically predicted response for measured quasi-static frame properties, resulting in bad correlation. In a second step, the properties of the poroelastic material are estimated by fitting the predicted results to the experimental results, and following the behaviour imposed by the physics of the augmented Hooke's law [17]. 


\section{Biot's equations for modelling of isotropic porous materials}

A three dimensional theory describing wave motion in fluid saturated porous media was given by Biot [23], and will only be shortly revisited here. It should be kept in mind that a weak form of these equations, solved using a numerical procedure allows for the prediction of the response of quite general trim packages including porous materials.

The theory proposed by Biot describes the motion of the porous material by means of the homogenised displacements of the fluid and solid phase in the material on a macroscopic level, represented by the vectors $\mathbf{u}^{\mathbf{f}}$ and $\mathbf{u}^{\mathbf{s}}$. The interaction between these two phases introduces a coupled wave motion in the material, i.e. the different waves propagate in both the fluid and the solid frame simultaneously [5]. Assuming a time dependence $\mathrm{e}^{\mathrm{j} \omega t}$, with $\mathrm{j}^{2}=-1$, $\omega$ the angular frequency and time $t$; the equations of motion for isotropic porous media derived from the Biot theory can be expressed as [23]:

$$
\begin{aligned}
-\omega^{2} \rho_{1} \mathbf{u}^{\mathbf{s}} & -\left(\omega^{2} \rho_{\mathrm{a}}-\mathbf{j} \omega b(\omega)\right)\left(\mathbf{u}^{\mathbf{s}}-\mathbf{u}^{\mathbf{f}}\right) \\
& =(A(\omega)+N(\omega)) \nabla \nabla \cdot \mathbf{u}^{\mathbf{s}}+N(\omega) \nabla^{2} \mathbf{u}^{\mathbf{s}}+Q(\omega) \nabla \nabla \cdot \mathbf{u}^{\mathbf{f}}, \\
-\omega^{2} \rho_{2} \mathbf{u}^{\mathbf{f}} & -\left(\omega^{2} \rho_{\mathrm{a}}-\mathbf{j} \omega b(\omega)\right)\left(\mathbf{u}^{\mathbf{f}}-\mathbf{u}^{\mathbf{s}}\right) \\
& =R(\omega) \nabla \nabla \cdot \mathbf{u}^{\mathbf{f}}+Q(\omega) \nabla \nabla \cdot \mathbf{u}^{\mathbf{s}} .
\end{aligned}
$$

The interaction between the solid and the fluid phase can clearly be seen from these equations through the elastic coupling coefficient $Q(\omega)$, and the coefficients $\rho_{\mathrm{a}}$ and $b(\omega)$ related to the inertial and viscous coupling between both phases respectively. Note that several of the coefficients in Eqs. (1)-(2) are in general complex and frequency-dependent. The first term on the left- 
hand side in both equations considers the inertia of the solid (fluid) phase, with $\rho_{1}\left(\rho_{2}\right)$ the bulk density of the solid (fluid) phase. The second term on the left-hand side is directly proportional to the relative motion between the solid and the fluid phase and the dynamic coupling effects between both. The coupling phenomena related to viscosity are gathered in the term $b(\omega)$ and the interaction resulting from inertial forces is accounted for by the term $\rho_{a}$. The parameters $A(\omega), N(\omega), Q(\omega)$ and $R(\omega)$ define the stress-strain relations in the porous material, first derived by Biot [23], assuming no frequency dependence. Poroelastic materials used in vibro-acoustic treatments are often open cell, and the interstitial fluid is generally air. It may then be assumed that the bulk modulus of open-cell porous materials is several order of magnitude smaller than the bulk modulus of the constituent solid. Under this assumption, the parameters $A(\omega)$ and $N(\omega)$ are the first and second Lamé coefficients for the solid frame of the porous aggregate, $R(\omega)$ is the frequency-dependent bulk modulus of the fluid, and $Q(\omega)$ quantifies the dilatational coupling between both phases. The two latter parameters are functions of the porosity and the dynamic bulk modulus of the fluid [11], under the aforementioned assumption.

The coefficients in Eqs. (1)-(2), can be calculated from the 'geometrical' and 'structural' material parameters of the porous material. The parameters porosity $\phi$, flow resistivity $\sigma$, tortuosity $\alpha_{\infty}$ and the viscous and thermal characteristic lengths $\Lambda$ and $\Lambda^{\prime}$ are referred to as 'geometrical' parameters since they describe the behaviour of the fluid inside the porous material if the frame is motionless, in an equivalent fluid description, and hence are only 
related to the geometric structure of the porous material. The 'structural' parameters, Young's modulus $E$, Poisson's ratio $\nu$ and loss factor $\eta$ are bulk properties of the solid phase of the material.

The coupled equations of motion, Eqs. (1)-(2), describe three propagating waves in an isotropic porous material, two compressional waves and one shear wave. These three waves propagate simultaneously in both the fluid and the solid frame, however, the relative amplitude and phase between the resulting motion of both phases will be different. The shear wave propagates mainly in the solid phase, the associated fluid motion being a result of the interaction throughout the porous material. The compressional waves propagate in the fluid and solid phases simultaneously and may result in a strong coupling or decoupling between the solid and fluid phases in the frequency range of interest. The two compressional waves propagate through the material with a different velocity, the wave with highest propagation velocity being referred to as the fast wave, and the other one as the slow wave. The slow wave has a higher damping than the other waves, as the solid and fluid phase move out of phase, amplifying the dissipation effects which are proportional to the relative motion between both phases. For the fast wave and the shear wave, both phases are moving in phase, and these waves are thus less damped.

\section{Augmented Hooke's law}

The parameters defining the visco-elastic properties of a poroelastic ma-

terial are in general frequency-dependent, in particular if the frame is constituted of a polymeric material, which has its glass transition temperature at 
room temperature [24]. For materials showing such visco-elastic behaviour, it is important to take the frequency dependence of the frame stiffness into account.

The augmented Hooke's law (AHL) by Dovstam [16, 17], who proposed a linear, fully three-dimensional constitutive material damping formulation, uses the approach to viscoelasticity first initiated by Biot $[25,26]$. In the current paper, the isotropic form of the augmented Hook's law is chosen to describe the anelastic behaviour of the frame of the porous material. The augmented Hooke's law expresses the stiffness parameters of the material as a superposition of an elastic, frequency-independent part for the fully relaxed material deformation and an anelastic, frequency-dependent part for the reversible visco-elastic deformation. This separation was motivated by Biot $[25,26]$ who introduced the concept of hidden thermodynamic variables, and described the dynamic elastic moduli of the solid as a superposition of elastic and anelastic contributions. For the details on the Augmented Hooke's Law, the reader is referred to the original papers by Dovstam $[16,17,27]$.

The isotropic model of the Augmented Hooke's Law is given by

$$
\begin{aligned}
& A(\omega)=\hat{A}\left(1+d_{\mathrm{A}}(\omega)\right), \\
& N(\omega)=\hat{N}\left(1+d_{\mathrm{N}}(\omega)\right),
\end{aligned}
$$

where $\hat{A}$ and $\hat{N}$ are the real-valued, static elastic Lamé moduli of the material, and $d_{\mathrm{A}}(\omega)$ and $d_{\mathrm{N}}(\omega)$ are complex frequency-dependent damping functions [27]. These two damping functions are not necessarily equal, however, 
they are chosen here to be identical, which results in a real-valued, frequencyindependent and constant Poisson's ratio. The real and imaginary part of the damping function represent the added dynamic stiffness due to stress-strain relaxation and the dissipation respectively;

$$
d_{\mathrm{A}}(\omega)=d_{\mathrm{N}}(\omega)=\sum_{l=1}^{N_{\mathrm{a}}} \frac{\mathrm{j} \omega}{j \omega+\beta_{l}} C_{l}
$$

with $\beta_{l}$ and $C_{l}$ the relaxation frequencies and amplitudes of each relaxation

process; and $N_{\mathrm{a}}$ the number of relaxation processes; and should generally be considered as a material parameter. Note that with the choice of parametrisation shown in Eqs. (3)-(5) the stated isotropic augmented Hooke's law corresponds to a frequency-dependent proportional damping model [16].

As a consequence of the chosen material model, i.e. the isotropic augmented Hooke's law with identical damping functions for both Lamé moduli, the real part of the moduli of the material will be equal to their static value at $0 \mathrm{~Hz}$, and will strictly increase with frequency and reach a high frequency limit for infinite frequencies. The imaginary part of the moduli, representing the material dissipation, will be equal to zero at $0 \mathrm{~Hz}$ and will approach zero for infinite frequencies, and must pass through at least one maximum, although it is possible that more maxima exist, which is also concluded by Pritz [19, 28, 29] from measurements on polymeric damping materials.

\section{Materials}

Two different materials are studied, both used in automotive applications. Material A is an elastic material with limited visco-elasticity. Material B is 
Table 1: Material parameters as given by manufacturer.

\begin{tabular}{ccccc}
\hline & Property & plate & $\mathrm{A}$ & $\mathrm{B}$ \\
\hline$t$ & Thickness [m] & 0.0014 & 0.020 & 0.020 \\
$\sigma$ & Air flow resistivity [Pa.s $\left./ \mathrm{m}^{2}\right]$ & - & 32950 & 80650 \\
$\phi$ & Porosity [-] & - & 0.95 & 0.94 \\
$\alpha_{\infty}$ & Tortuosity [-] & - & 2.1 & 2.5 \\
$\lambda$ & Viscous characteristic length $[\mu \mathrm{m}]$ & - & 50 & 27 \\
$\lambda^{\prime}$ & Thermal characteristic length $[\mu \mathrm{m}]$ & - & 150 & 81 \\
$\rho_{1}$ & Bulk density [kg/m $\left.{ }^{3}\right]$ & 7663 & 55 & 63 \\
$E$ & Young's Modulus [Pa] & $217 \mathrm{E} 9$ & 20203 & 41434 \\
$\eta$ & Loss factor [-] & 0.002 & 0.21 & 0.29 \\
$\nu$ & Poisson ratio [-] & 0.30 & 0.25 & 0.20 \\
\hline
\end{tabular}

a highly visco-elastic material. The parameters of both materials have been provided by the manufacturer. The Young's modulus, loss factor and Poisson ratio were measured by the manufacturer with a quasi-static method at low frequency $(30 \pm 5 \mathrm{~Hz})$ according to the procedure described in [30,31], and provide the starting point for the current work. Table 1 gives the parameters for the two materials studied, and for the steel plate. 


\section{Measurements of coated steel plate panel}

\subsection{Experimental set-up}

The measurements are performed on a set-up designed to cover a wide range of structure-trim interaction phenomena present in automotive applications [32]. A steel plate sample is clamped in a rigid frame which is excited by a shaker-stinger connection with a uniform boundary acceleration, normal to the test panel, seen in Figure 1. The unidirectional acceleration normal to the test panel is assured by carefully installed air bearings and air springs. The frequency range of interest varies from $30 \mathrm{~Hz}$ until $1 \mathrm{kHz}$. The dimensions of the test samples are $350 \mathrm{~mm} \times 500 \mathrm{~mm}$, and the set-up allows for a variable plate thickness to be used. A detailed description of the set-up is given by Van der Kelen et al. [32]. Its main features are shortly reviewed here for completeness and understanding.

The set-up is designed with the expressed purpose of realising a reproducible and easy to model boundary condition of a steel plate with trim configuration. A steel panel of thickness $1.4 \mathrm{~mm}$ is clamped between a lower and upper rigid frame, pulled together by 62 equidistant bolts. A preliminary study of the clamping conditions by comparing the experimental to predicted results, showed that a sufficiently good clamping was achieved by applying a torque of $10 \mathrm{Nm}$ to each bolt.

When the upper frame used for the clamping is placed on the test panel, it creates a cavity of dimensions $350 \mathrm{~mm} \times 500 \mathrm{~mm} \times 15 \mathrm{~mm}$ in which the trim package to be tested can be installed. This cavity will be referred to as 


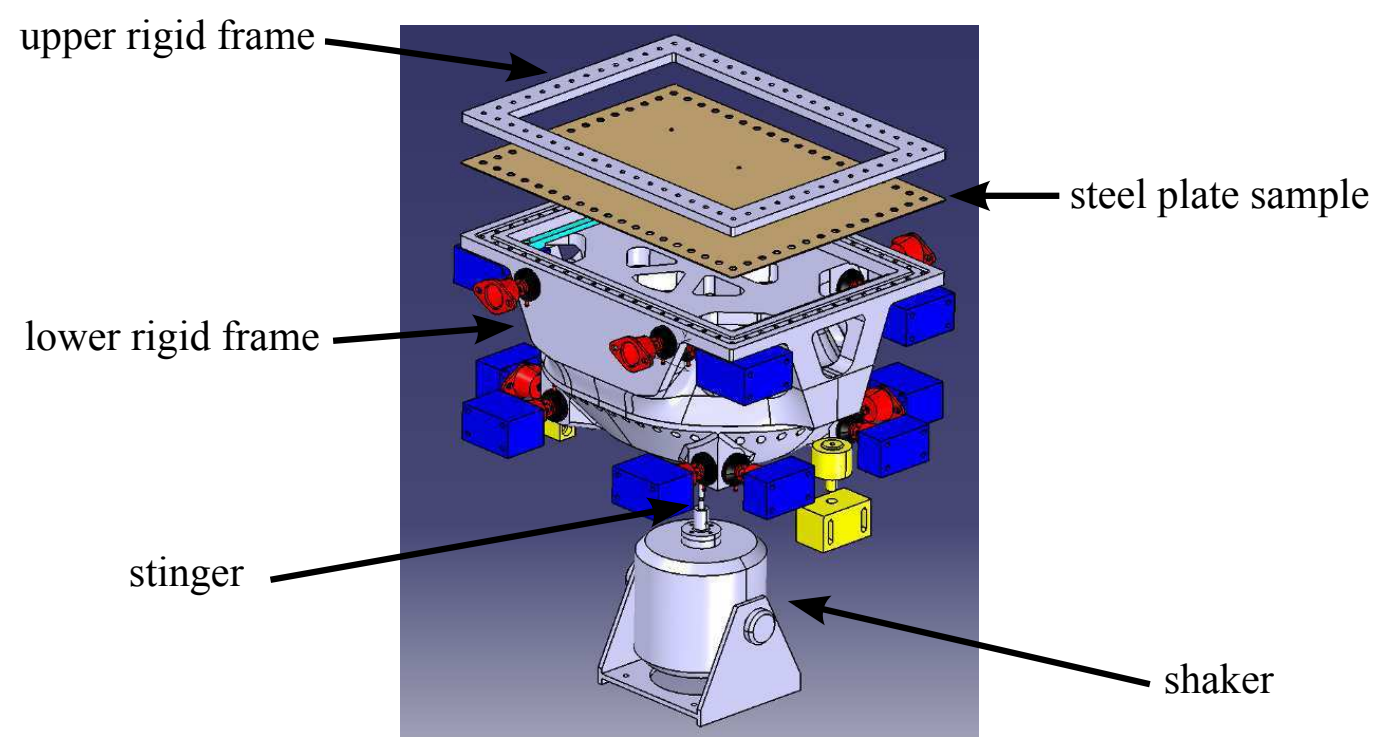

Figure 1: Schematic representation of the set-up, brown panel: steel plate sample, blue elements: air bearings, yellow elements: air springs.

the trim cavity. The porous materials tested here, however, have dimensions $350 \mathrm{~mm} \times 500 \mathrm{~mm} \times 20 \mathrm{~mm}$. The sides of the porous material are then in contact with the upper frame up till a height of $15 \mathrm{~mm}$, and the remaining $5 \mathrm{~mm}$ are in contact with the surrounding air. In the configuration investigated, the upper surface of the material is fully in contact with air as well.

The acceleration of the frame is measured with an accelerometer in a reference point on the frame, which was extensively investigated to give a good representation of the input excitation to the plate. A second accelerometer is placed in the middle of the steel panel (with or without coating). The Frequency Response Function (FRF) between the measured responses is then calculated, giving the acceleration of the middle of the steel panel over the 
input excitation to the plate. The signal to the shaker, which excites the steel panel is a periodic logarithmic chirp from 50 to $1300 \mathrm{~Hz}$. The experimental set-up was checked for linearity and repeatability, which both proved to be satisfactory.

\subsection{Influence of adhesive on plate response}

Both porous materials were fastened to the plate with double-sided adhesive, in order to approximate a bonded plate-porous material boundary condition. Before measuring each material, the effect of the adhesive on the plate response was studied, as a quality control of the measured data. Table 2 gives the measured resonance frequencies of the plate without and with adhesive for 3 repeated measurements. The layer of adhesive was removed and another layer was mounted in between the measurements. The effect of the adhesive on the acceleration of the plate is small, and different for each mounted layer of adhesive, and the adhesive is found to mostly lower the eigenfrequencies. Note that, due to the symmetric excitation in the measurement set-up, only the odd plate modes are excited, as shown in Table 2.

\subsection{Measured response of the coated panel}

Figure 2 shows the transfer function of the acceleration measured in the middle of the plate relative to the input acceleration, for the plate without coating, and coated with materials A and B. The porous materials are glued onto the plate with adhesive to approximate a bonded plate-porous material boundary condition. There is no treatment applied to the sides of the porous material, however the material fits snugly into the trim cavity made by the 
Table 2: Modes and measured resonance frequencies of the plate (frequencies in $\mathrm{Hz}$ ) and the influence of the used adhesive.

Mode $\left(n_{\mathrm{x}}, n_{\mathrm{y}}\right) \quad$ without adh. adh. config. 1 adh. config. 2 adh. config. 3

\begin{tabular}{lllll}
\hline$(1,1)$ & 79.38 & 78.75 & 81.88 & 81.56 \\
$(3,1)$ & 201.25 & 199.38 & 203.75 & 201.88 \\
$(1,3)$ & 367.5 & 363.44 & 368.13 & 366.88 \\
$(5,1)$ & 452.5 & 448.44 & 452.81 & 449.06 \\
$(3,3)$ & 483.44 & 479.06 & 483.13 & 481.25 \\
$(5,3)$ & 720.94 & 714.69 & 719.06 & 715.00 \\
$(7,1)$ & 820.31 & 814.69 & 818.13 & 814.38 \\
$(1,5)$ & 894.69 & 886.88 & 890.63 & 885.63 \\
$(3,5)$ & 1010.0 & 1001.9 & 1004.7 & 1000.6 \\
\hline
\end{tabular}

upper rigid frame. The response is measured from 100 to $1000 \mathrm{~Hz}$, with a frequency resolution of $0.3125 \mathrm{~Hz}$. Both the measured amplitude and phase are shown in the figure.

The effect the two materials have on the response of the plate is significant. Both materials have, according to the manufacturers' data, a comparably low Young's modulus, high mass density and high loss factors. The influence of materials $\mathrm{A}$ and $\mathrm{B}$ is very similar up to $600 \mathrm{~Hz}$, forcing the resonances to lower frequencies as well as dampening the response. Material A results in a slightly more damped response than material B around $400 \mathrm{~Hz}$. At higher frequencies, material B gives a considerably more heavily damped response. 

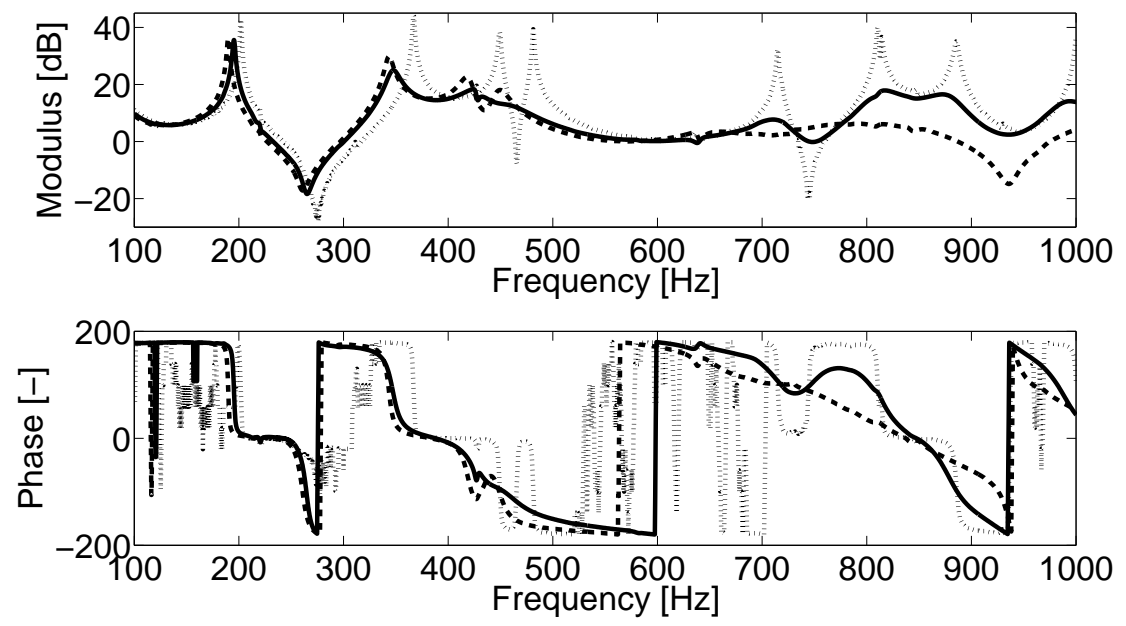

Figure 2: Comparison of measured response in the middle of the plate with and without porous material, amplitude and phase - dotted line: plate without coating; solid line: plate coated with material A; dashed line: plate coated with material B.

\section{Prediction of response of coated panel with frequency-dependent elastic parameters}

\subsection{Numerical models of poroelastic materials}

A numerical model of the set-up with linear or quadratic elements quickly leads to a prohibitively large amount of degrees of freedom $(\mathrm{DOFs})(\approx$ millions). In addition to resulting in excessive calculation times, the convergence of the model is difficult to assure. This is a particular difficulty in the numerical solution of 3D models including porous materials as the convergence criteria for these models are different from the classical criteria, used in finite element modelling $[7,9]$.

Steps towards solving 3D models with porous materials were made by 
Hörlin et al. [8, 10] and Rigobert et al. [9] with finite element schemes for three-dimensional poroelastic materials using hierarchical elements. Hierarchical elements have the advantage to allow for higher-order polynomial basis functions, and a more accurate representation of the different types of deformations while still retaining coarse meshes, as compared to classical finite elements.

The hierarchical $h p$-FEM used in this work is an in-house finite element code here using the implemented mixed pressure-displacement formulation with the solid displacement and the fluid pressure as dependent variables. This mixed displacement-pressure formulation, first derived by Atalla et al. [33] has the advantage of reducing the number of degrees of freedom per node from six to four. Different interpolation orders can be used for the basis functions of each phase separately, as well as in the different Cartesian coordinate directions, potentially leading to a significant reduction of the number of degrees of freedom required for a correct modelling, and hence a reduction in computational cost. The code used allows for an AHL damping description of the solid frame to be included in the model. A detailed description of the numerical prediction tool used, is out of the scope of this paper, and can be found in $[8,10,34]$.

\subsection{Modelling of the set-up}

The set-up is modelled consisting of three parts, as shown in Figure 3. The plate is modelled as a parallelepiped-shaped domain with dimensions 0.5 $\mathrm{m} \times 0.35 \mathrm{~m} \times 0.0014 \mathrm{~m}$. The porous material coating the plate is modelled as a parallelepiped-shaped domain with dimensions $0.5 \mathrm{~m} \times 0.35 \mathrm{~m} \times 0.020$ 


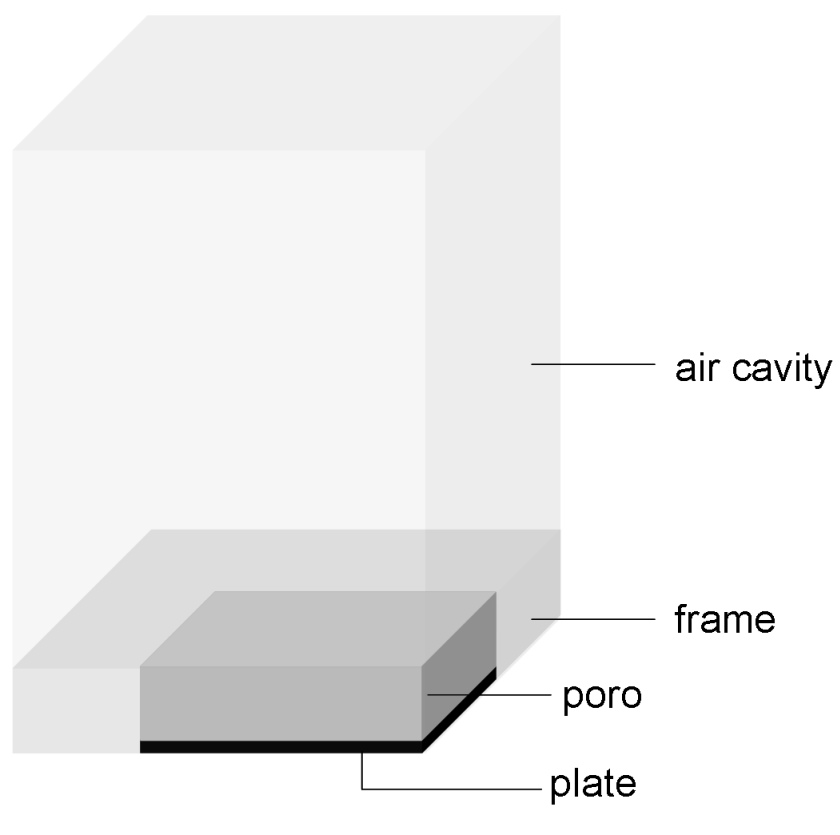

Figure 3: Representation of a quarter of the full set-up, as the geometry is modelled in the Finite Element model, using the symmetry of the set-up. 
$\mathrm{m}$, and is in contact with the solid frame at its boundaries. In contrast to the experimental set-up, the frame has the same height as the porous material, is $0.05 \mathrm{~m}$ wide and enclosing the porous domain. An air cavity has been modelled on top of the set-up to simulate the contact with air, using a parallelepiped-shaped domain with dimensions $0.6 \mathrm{~m} \times 0.45 \mathrm{~m} \times 0.7 \mathrm{~m}$. The presence of this air cavity has no influence on the predicted response of the plate. The boundary condition between the plate and the porous domain is assumed to be bonded. The sides of the porous domain are bonded to the solid frame, an assumption representing the boundary condition between the porous material and the frame used to clamp the plate. The discrepancy with the experimental set-up, where the upper $5 \mathrm{~mm}$ of porous material is in contact with air instead of the solid frame, due to the difference in height of the trim cavity and the thickness of the tested materials (see Section 5.1), is neglected, and is assumed to have a minimal effect on the results studied here. The upper surface of the material is in contact with air as is the case in the measurements. The excitation of the plate is a prescribed displacement along the boundaries, with an amplitude of $0.001 \mathrm{~m}$. The symmetry of the set-up and the excitation allowed for modelling a quarter of the full set-up.

The number of elements and the order of the polynomials used for the plate, the poroelastic material and the air cavity are given in Table 3. Note that an anisotropic p-extension is used in the thickness and the in-plane directions, and that the basis functions for both phases of the porous material have the same interpolation orders. 
Table 3: Number of elements and polynomial order of different domains.

\begin{tabular}{c|cc|cc} 
domain & \multicolumn{2}{|c|}{ elements } & \multicolumn{2}{c}{ polynomial order } \\
\hline & thickness & in-plane & thickness & in-plane \\
\hline plate & 1 & 1 & 2 & 9 \\
cavity & 4 & 1 & 9 & 9 \\
porous material & 1 & 1 & 3 & 9 \\
\hline
\end{tabular}

\section{Correlation between measured and simulated results}

\subsection{Steel panel without porous material}

Figure 4 shows a comparison of the measured and predicted amplitude of the FRF of the plate without coating (full line identical to dotted line in Figure 2). A very good agreement of the levels and good agreement of the resonance frequencies may be observed. The maximum deviation in resonance frequency is $1.4 \%$ for mode $(3,5)$ at $996 \mathrm{~Hz}$. Towards higher frequencies, there is a small discrepancy between measured and simulated responses. An imperfect clamping of the plate or the mesh density used in the prediction may be plausible reasons for the discrepancy. Note that the difference between the measured and simulated response is not larger than the differences between the different adhesive configurations, Table 2. The influence of the adhesive will not be taken into account in the further discussions.

\subsection{Steel plate panel coated with material A}

Figure 5 shows that the overall trend in the measured responses for a point in the middle of the plate with porous material $\mathrm{A}$ is predicted to a 


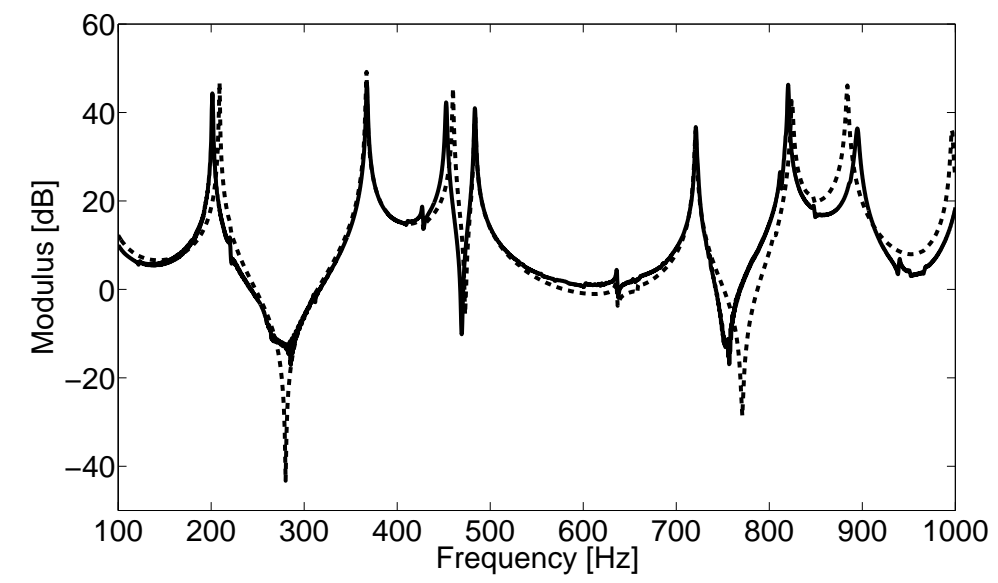

Figure 4: Comparison of measured and predicted response in the middle of the plate for the plate without coating - full line: measurement (identical to dotted line in Figure 2); dashed line: prediction.
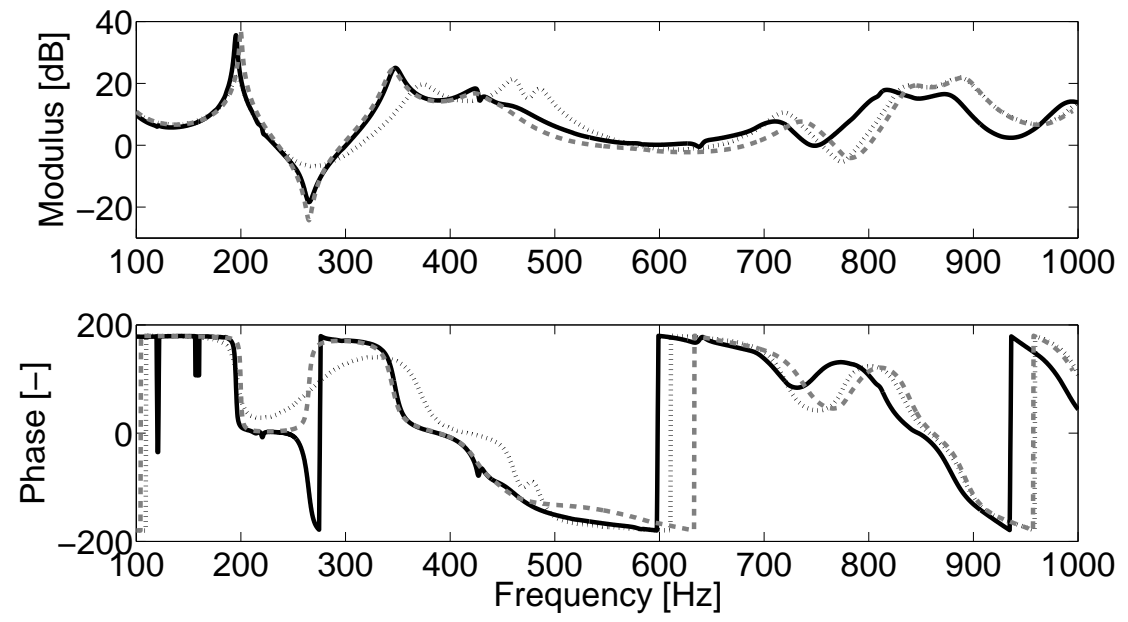

Figure 5: Comparison of measured and predicted response in middle of the plate coated with material A, amplitude and phase - black solid line: measurement; black dotted line: prediction with nominal constant elastic parameters; grey dashed line: prediction with constant modulus, equal to four times the nominal value measured, see Table 1. 

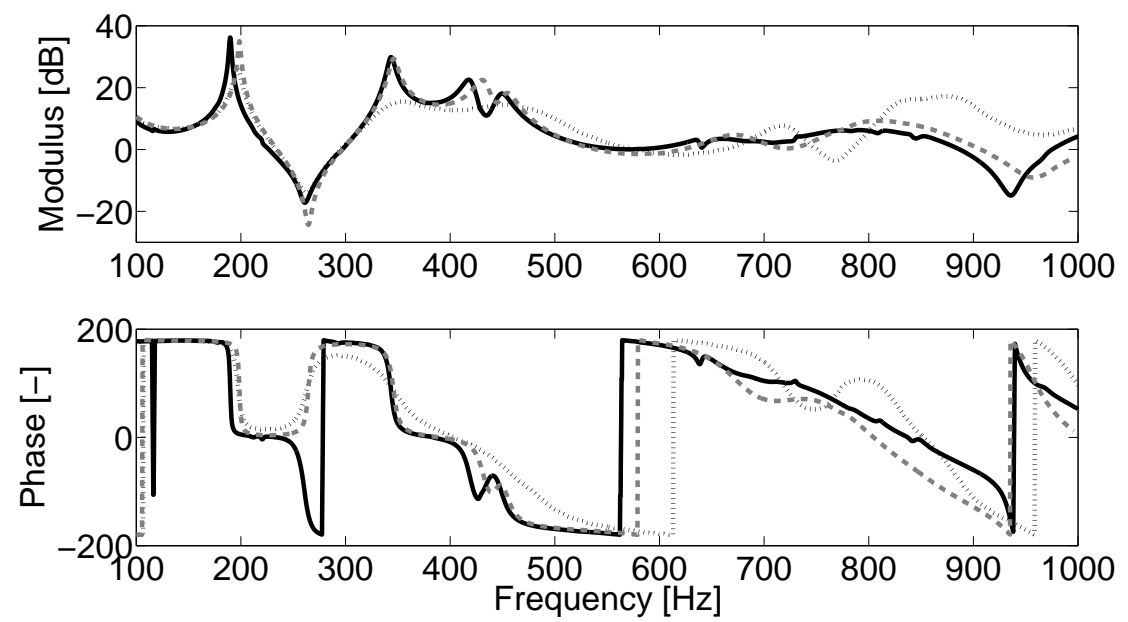

Figure 6: Comparison of measured and predicted response in middle of the plate coated with material B, amplitude and phase - black solid line: measurement; black dotted line: prediction with constant nominal elastic parameters; grey dashed line: prediction with AHL model.

certain degree of accuracy with the given, frequency-independent, stiffness properties (black dotted line). However, between 200 and $600 \mathrm{~Hz}$, the resonance peaks are shifted to higher frequencies.

\subsection{Steel plate panel coated with material B}

From Figure 6, it is clear that the responses measured in the middle of the plate and the responses predicted with porous material B with constant stiffness properties (black dotted line) do not agree particularly well. Between $300 \mathrm{~Hz}$ and $600 \mathrm{~Hz}$, the predicted response is underestimating the measured response, while for higher frequencies, the opposite appears to be true. 


\section{Correlation between measurement and simulated results, ac- counting for the dynamic material behaviour}

\subsection{Estimation of dynamic material behaviour}

In order to investigate the sensitivity of the predicted response to possible uncertainties in the input parameters related to the elastic properties, simulations were repeated with values for the Young's modulus and loss factor which differ from the values in Table 1. A better correlation between predictions and experiments was obtained by evaluating different Young's moduli in a number of small frequency ranges, following the physics in the augmented Hooke's law, which requires a strict increase of the Young's modulus with frequency, as described in section 3. The Young's modulus and loss factor for which a good correlation was obtained between the prediction and measurement in the selected small frequency range, was then assumed to be a better representation of the Young's modulus and loss factor at the centre point of this small frequency range. When applicable, an augmented Hooke's law damping model approaching this behaviour was then fitted using the procedure explained in [35].

\subsection{Results}

\subsubsection{Steel plate panel coated with material $A$}

The best correlation between measurement and simulation for the steel plate coated with material A was for a Young's modulus which is constant and equal to four times the value given in Table 1 over the studied frequency range, as shown in Figure 5 (grey dashed line). Increasing the modulus has a clear effect on the response predicted up to about $600 \mathrm{~Hz}$. The resonance 
peaks are shifted towards lower frequencies, which may be caused by the interaction between the steel plate and the poroelastic material in this frequency range. The shift to higher frequencies has also been reported by Litwinczik et al. [2]. Above $600 \mathrm{~Hz}$, the predicted responses are similar but shifted in frequency in both amplitude and phase compared to the experimental results. This implies that the influence of a variation in the Young's modulus at higher frequencies is moderate and the discrepancies observed must be due to other parameters or differing conditions between the simulation model and the physical set-up. The increase in the Young's modulus is according to the physics in the augmented Hooke's law, however it is important to note that a frequency dependent Young's modulus is not considered for material A, since the results suggest that the asymptotic high frequency value of the Young's modulus has been reached already at $100 \mathrm{~Hz}$, which is the lowest frequency present in the measured response.

\subsubsection{Steel plate panel coated with material B}

For material B, the best correlation between measurement and simulation was obtained by fitting an Augmented Hooke's law damping model through the different values obtained. The result was a frequency-dependent Young's modulus and damping function as presented in Figure 7 (grey dashed line). The correlation between measured and predicted response is substantially improved when the frequency dependence of properties of the solid frame is taken into account. Below $500 \mathrm{~Hz}$, the predicted amplitude agrees better with the measured amplitude and is less damped, and the peaks near 400 $\mathrm{Hz}$ are now shifted closer to the measured frequencies, in agreement with the observations by Litwinczik et al. [2] and in Figure 5. At higher frequencies, 


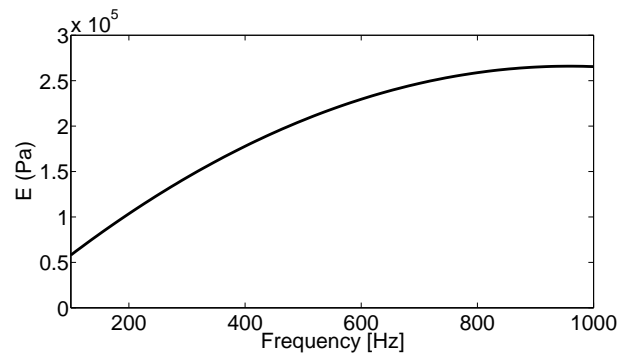

(a) Frequency-dependent Young's modulus (Real part)

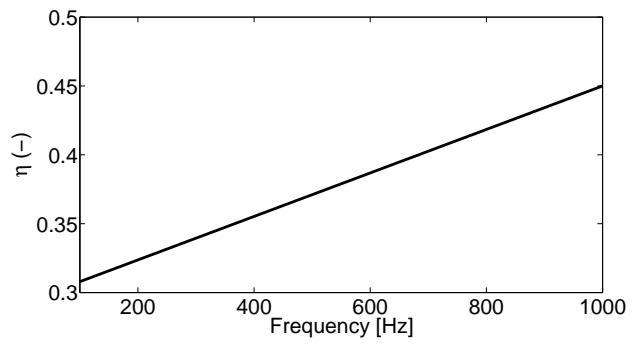

(b) Frequency-dependent loss factor

Figure 7: Fitted frequency dependence of Young's modulus (real part) and loss factor for material B.

the response is highly influenced by the increase in Young's modulus, and is now highly damped, suggesting there is a particular interaction taking place between the plate and the porous material at these frequencies. In addition, the predicted response is very sensitive in this frequency range to variations in the Young's modulus, encumbering a better correlation. The assumptions related to the boundary conditions of the porous material may also prohibit a better fit, as the response may be more sensitive to their influence. 

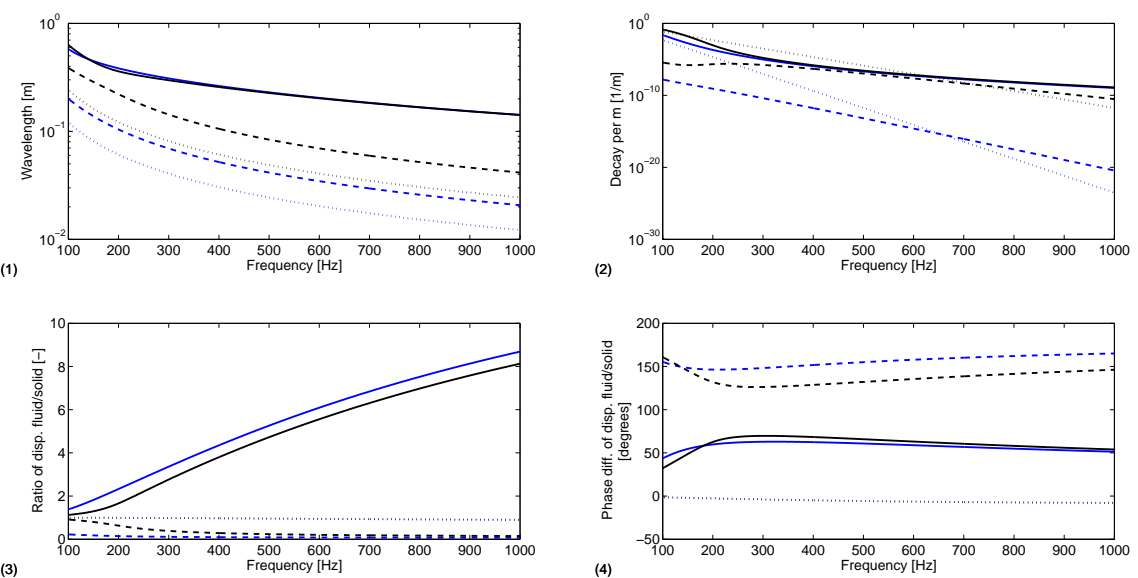

(a) Wave characteristics of material A.
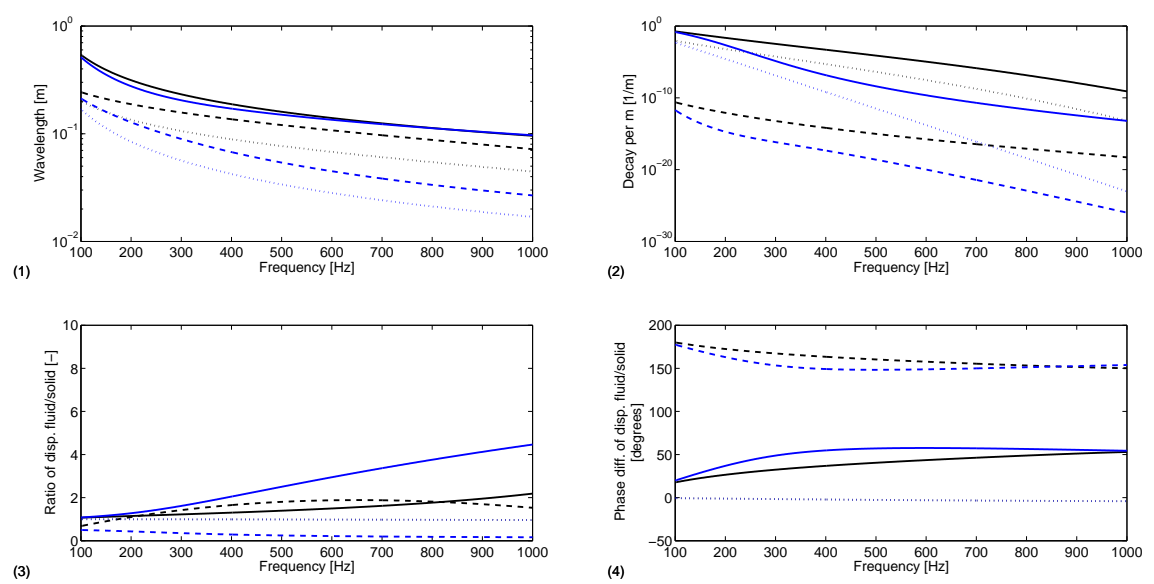

(b) Wave characteristics of material B.

Figure 8: Fast compressional wave (solid line), slow compressional wave (dashed line) and shear wave (dotted line), characteristics for given elastic parameters (blue) and for estimated material parameters (black), (1) wavelength $[\mathrm{m}],(2)$ decay per $\mathrm{m}[1 / \mathrm{m}]$ (the decay rate is close to one for a lowly damped wave [5]), (3) ratio of fluid over solid displacement [-], (4) phase difference of fluid over solid displacement [degrees]. Note that the graphs for the shear wave (blue and black dotted line) in panels 3 and 4 are on top of each other. 


\section{Influence of estimated elastic parameters on material behaviour}

It is interesting to study some characteristic features for the three Biot waves in both materials, both for the measured, frequency-independent material parameters and for the estimated material parameters. These are shown in Figure 8 for materials A and B. The wave characteristics of both materials for the measured properties (see Table 1) appear to be similar. The fast wave in both materials is the wave which has a preference for propagating in the fluid, as may be seen from the ratio of fluid/solid displacement, Figures 8a(3) and $8 \mathrm{~b}(3)$. The slow wave on the other hand, has a preference for propagating in the solid phase of the material. In both materials, the fluid and solid displacement, related to the compressional waves, are not exactly in or out of phase, Figures $8 \mathrm{a}(4)$ and $8 \mathrm{~b}(4)$, indicating that the solid and fluid phases are coupled due to the wave propagation in the frequency range of interest.

When studying the characteristics of the material for the estimated properties, the fast and the slow wave may still be distinguished, although the difference between the wavelengths is smaller. The damping levels of the slow wave and in particular the shear wave are highly affected at higher frequencies. Because of the bending in the plate, the stronger presence of the shear wave may possibly have a large effect on the plate response. For material B, the frequency-dependent stiffness properties of the material also strongly affect the preference of the compressional slow wave to propagate in the solid phase. Both waves are almost equally present in both phases. These results suggest that the frequency dependence of the stiffness parameters of the porous material, here fitted to the measured response of the coated plate, 
has a large influence on its characteristic features.

\section{Conclusion}

This paper presents new measurements of a steel plate sample coated with two different porous materials A and B, which are used in automotive applications. The coated plate is clamped and excited on its boundaries in a dedicated set-up designed for this purpose. The poroelastic materials have a large influence on the measured acceleration of the steel plate, which is most pronounced at higher frequencies. Subsequently, the response of the coated panel for both poroelastic materials is predicted with an in-house hierarchical finite element code. The elastic materials parameters used for these predictions were provided from quasi-static measurements performed by the material supplier. For material A, the predicted response is similar to the measured response to a certain extent. For material B, a significant discrepancy is found between the measured and predicted response, which is more pronounced at high frequencies.

A hierarchical $h p$-FE code, which allows for an augmented Hooke's law damping model to be included, is used to here as a tool to obtain a better correlation between the measured and predicted responses. The augmented Hooke's law, which may used to describe the viscoelastic behaviour of poroelastic materials, served as a guideline to capture the physical behaviour of the studied materials. The stiffness parameters of the material were modified after recalculating the predicted FRF for a set of elastic parameters differ-

ing from the measured ones, following the physics in the augmented Hooke's 
Law, and this resulted in a remarkable improvement in correlation in the studied frequency range for both materials. For material A, it seems that the asymptotic value at high frequency is reached at $100 \mathrm{~Hz}$. For material B, a frequency-dependent augmented Hooke's Law is estimated in the studied frequency range.

These results emphasise the necessity of characterising the dynamic behaviour of porous materials, and indicate the augmented Hooke's law as an interesting model for capturing the viscoelastic behaviour of poroelastic materials. The model may thus be used as well in developing material characterisation methods which can account for the visco-elastic material behaviour, e.g. $[35,36]$, in particular in configurations and applications where they are predominantly excited structurally. 


\section{Acknowledgements}

The IWT Flanders and the Fund for Scientific Research - Flanders (F.W.O.) are gratefully acknowledged for their support. The authors wish to thank Mr. Kobayashi from Toyota Motor Corporation, and Dr. Bergen and Mr. Schaefer from Toyota Motor Europe for their support in performing the experimental work.

[1] S. Rigobert, F. C. Sgard, and N. Atalla. A two-field hybrid formulation for multilayers involving poroelastic, acoustic, and elastic materials. Journal of the Acoustical Society of America, 115:2786-2797 (2004).

[2] V. Litwinczik, W. Lauriks, A. Lenzi, G. Geentjens. Radiation efficiency of a simply supported covered plate: prediction and measurement. Proceedings International Congress on Sound and Vibration ICSV, (2005).

[3] O. Doutres, N. Dauchez. Characterization of porous materials viscoelastic properties involving the vibroacoustical behavior of coated panels. Proceedings of SAPEM 05, (2005).

[4] L. Jaouen, B. Brouard, N. Atalla, C. Langlois. A simplified numerical model for a plate backed by a thin foam layer in the low frequency range. Journal of Sound and Vibration, 280:681-698 (2005).

[5] P. Göransson. Acoustic and vibrational damping in porous solids. Philosophical Transactions of the Royal Society A: Mathematical, Physical and Engineering Sciences, 364:89-108 (2006). 
[6] O. Doutres, N. Dauchez, J.-M. Génevaux, and O. Dazel. Validity of the limp model for porous materials: A criterion based on the Biot theory. Journal of the Acoustical Society of America, 122:2038-2048 (2007).

[7] N. Dauchez, S. Sahraoui, and N. Atalla. Convergence of poroelastic finite elements based on Biot displacement formulation. Journal of the Acoustical Society of America, 109:33-40 (2001).

[8] N.-E. Hörlin, M. Nordström, and P. Göransson. A 3-D hierarchical FE formulation of Biot's equations for elasto-acoustic modelling of porous media. Journal of Sound and Vibration, 245:633-652 (2001).

[9] S. Rigobert, N. Atalla, and F. C. Sgard. Investigation of the convergence of the mixed displacement-pressure formulation for three-dimensional poroelastic materials using hierarchical elements. Journal of the Acoustical Society of America, 114:2607-2617 (2003).

[10] N.-E. Hörlin. 3D hierarchical $h p$-FEM applied to elasto-acoustic modelling of layered porous media. Journal of Sound and Vibration, 285:341$363(2005)$.

[11] J. F. Allard and N. Atalla. Propagation of Sound in Porous Media: Modelling Sound Absorbing Materials. John Wiley \& Sons, 2nd edition, 372 pages (2009).

[12] R. Panneton and X. Olny. Acoustical determination of the parameters governing viscous dissipation in porous media. Journal of the Acoustical Society of America, 119:2027-2040 (2006). 
[13] X. Olny and R. Panneton. Acoustical determination of the parameters governing thermal dissipation in porous media. Journal of the Acoustical Society of America, 123:814-824 (2008).

[14] Y. Atalla and R. Panneton. Inverse acoustical characterization of open cell porous media using impedance tube measurements. Canadian Acoustics - Acoustique Canadienne, 33:11-24 (2005).

[15] T. Pritz. Transfer function method for investigating the complex modulus of acoustic materials: spring-like specimen. Journal of Sound and Vibration, 72:317-341 (1980).

[16] K. Dovstam. Augmented Hooke's law in frequency domain. A three dimensional, material damping formulation. International Journal of Solids and Structures, 26:2835-2852 (1995).

[17] K. Dovstam. Augmented Hooke's law based on alternative stress relaxation models. Computational mechanics, 26:90-103 (2000).

[18] T. Pritz. Measurement methods of complex Poisson's ratio of viscoelastic materials. Applied Acoustics, 60:279-292 (2000).

[19] T. Pritz. Five-parameter fractional derivative model for polymeric damping materials. Journal of Sound and Vibration, 265:935-952 (2003).

[20] W. Lauriks, L. Boeckx, P. Leclaire,P. Arora Nee Khurana,L. Kelders. Characterization of porous acoustic materials . Proceedings of SAPEM 05, (2005). 
[21] L. Jaouen, A. Renault, M. Deverge. Elastic and damping characterizations of acoustical porous materials: Available experimental methods and applications to a melamine foam. Applied Acoustics, 69:1129-1140 (2008).

[22] O. Doutres, N. Dauchez, J.-M. Genevaux, G. Lemarquand and S. Mezil. Ironless transducer for measuring the mechanical properties of porous materials. Review of Scientific Instruments, 81:055101 (2010).

[23] M. A. Biot. Theory of propagation of elastic waves in a fluid-satured porous solid. 1. low-frequency range. Journal of the Acoustical Society of America, 26:168-178 (1956).

[24] L. J. Gibson and M. F. Ashby. Cellular Solids: Structure and Properties. Cambridge University Press, 510 pages (1997).

[25] M. A. Biot. Theory of Stress-Strain Relations in Anisotropic Viscoelasticity and Relaxation Phenomena. Journal of the Acoustical Society of America, 25:1385-1391 (1954).

[26] M. A. Biot. Variational principles in irreversible thermodynamics with application to viscoelasticity. Physical Review, 97:1463-1469 (1955).

[27] K. Dovstam. Receptance model based on isotropic damping functions and elastic displacement modes. International Journal of Solids and Structures, 34:2733-2754 (1997).

[28] T. Pritz. Dynamic Young's modulus and loss factor of floor covering materials. Applied Acoustics, 49:179-190 (1996). 
[29] T. Pritz. Frequency dependences of complex moduli and complex Poisson's ratio of real solid materials. Journal of Sound and Vibration, 214:83-104 (1998).

[30] M. Melon, E. Mariez, C. Ayrault, and S. Sahraoui. Acoustical and mechanical characterization of anisotropic open-cell foams. Journal of the Acoustical Society of America, 104:2622-2627 (1998).

[31] C. Langlois, R. Panneton, and N. Atalla. Polynomial relations for quasi-static mechanical characterization of isotropic poroelastic materials. Journal of the Acoustical Society of America, 110:3032-3040 (2001).

[32] C. Van der Kelen, M. Vivolo, B. Van Genechten, B. Pluymers, W. Desmet, A. Malkoun, B. Bergen, and T. Keppens. Validation of a dedicated test set-up for boundary excitation of trim assemblies. Proceedings of ISMA2012, Leuven, Belgium, 2012 September 17-19, Leuven (2012).

[33] N. Atalla and R. Panneton. Enhanced weak integral formulation for the mixed (u, p) poroelastic equations. Journal of the Acoustical Society of America, 109:3065-3068 (2001).

[34] N. E. Hörlin and P. Göransson. Weak, anisotropic symmetric formulations of Biot's equations for vibro-acoustic modelling of porous elastic materials. International Journal for Numerical Methods in Engineering, 82:1519-1540 (2010).

[35] J. Cuenca, C. Van der Kelen, and P. Göransson. A general methodology for inverse estimation of the elastic and anelastic properties of 
anisotropic open-cell porous materials - with application to a melamine foam. Journal of Applied Physics, 115:084904 (2014).

[36] J. Cuenca and P. Göransson. Inverse estimation of the elastic and anelastic properties of the porous frame of anisotropic open-cell foams. Journal of the Acoustical Society of America, 132:621-629 (2012). 